\title{
Geophysical methods for the study of sedimentary cycles
}

\author{
Xu Jingling1, 2, Liu Luofu ${ }^{1,2 *}$, Wang Guiwen ${ }^{1,2}$, Shen Jinsong1,2 and \\ Zhang Chunhua ${ }^{3}$ \\ ${ }^{1}$ School of Resources and Information Technology, China University of Petroleum, Beijing 102249, China \\ ${ }^{2}$ State Key Laboratory of Petroleum Resource and Prospecting, China University of Petroleum, Beijing 102249, China \\ ${ }^{3}$ Department of Computer Science and Technology, China University of Petroleum, Beijing 102249, China
}

\begin{abstract}
We present the wavelet depth-frequency analysis and variable-scale frequency cycle analysis methods to study sedimentary cycles. The spectrum analysis, variable-scale frequency cycle analysis, and wavelet depth-frequency analysis methods are mainly discussed to distinguish sedimentary cycles of different levels. The spectrum analysis method established the relationship between the spectrum characteristics and the thickness and number of sedimentary cycles. Both the variable-scale frequency cycle analysis and the wavelet depth-frequency analysis are based on the wavelet transform. The variable-scale frequency cycle analysis is used to obtain the relationship between the periodic changes of frequency in different scales and sedimentary cycles, and the wavelet depth-frequency analysis is used to obtain the relationship between migration changes of frequency energy clusters and sedimentary cycles. We designed a software system to process actual logging data from the Changqing Oilfield to analyze the sedimentary cycles, which verified the effectiveness of the three methods, and good results were obtained.
\end{abstract}

Key words: Sedimentary cycles, spectrum analysis, wavelet depth-frequency analysis, variable-scale frequency cycle analysis

\section{Introduction}

Sedimentary strata are not only layered but also have cyclicity. The complex periodic movements in nature are the superposition of a variety of simple movements with different periods, and sedimentary cycles are the periodic repetition of sedimentary events (Wang et al, 2002; Li et al, 2003). Logging sequences of strata associated with contributing factors are the material records of a variety of sedimentary events within a specific time series, which can reflect the sedimentary characteristics of strata such as cyclicity and periodicity sensitively and continuously. Then, how can we identify sedimentary cycles from the observed data? In order to obtain more comprehensive understanding of the earth, geophysics must be combined with geology (Liu, 1998), which has become a more commonly used trend.

Some researchers used Fourier transform, short-time Fourier transform and simple wavelet transform to study sedimentary cycles and obtained good results (Li, 2006; Zhao and Li, 2008; Fang et al, 2007a). However, the previous studies simply relied on the rhythm of frequency to distinguish sedimentary cycles macroscopically using seismic data whose resolution was not high, and did not solve: (1) what standard scale can be used to distinguish

*Corresponding author. email: liulf@cup.edu.cn Received July 13, 2008 sedimentary cycles, (2) how to quantify the scale, (3) how to distinguish high-frequency cycles of sedimentary reservoirs without cores and outcrops. To solve the above problems, we presented the wavelet depth-frequency analysis and variablescale frequency cycle analysis methods to study sedimentary cycles. Both methods were applied to well logging data from the Changqing Oilfield, northwest China, and the results were analyzed.

\section{Method}

Variable-scale frequency cycle and wavelet depthfrequency analysis methods are both based on the wavelet transform. The latter method uses two key techniques: frequency division multi-scale automated processing along layer and high-resolution section reconstruction. Frequency spectrum analysis, which is the basis of the two methods, is also an effective way to analyze sedimentary cycles. The three analysis methods of sedimentary cycles have the same geological foundation. According to the principle of sedimentology, the geological sequence body, namely sedimentary strata, is composed of a group of strata, whose succession sequence is periodic and whose formation causes are related to one another. A sequence body has the hierarchical structure, and the hierarchy is not continuous and is mutually contained. The geological body as the exploration target has multi-scale and cyclical characteristics (Chen et al, 2007; Liu and Fang, 2006), and the cyclicity is 
recorded in the logging signals, which are different from the spectrum of seismic waves along the depth. This conforms to the characteristics of the three geophysical methods on studying signals, i.e., identifying the differences. By using the three geological methods, the spectrum characteristics, the change rules of variable-scale frequency, and the migration changes of frequency energy spectrum clusters, which are all corresponding to the sedimentary cycles, are extracted from logging signals (Peng, 1999; Fang et al, 2007b), to establish one-to-one mapping relationships between the geophysical data processing results and the characteristics of geological cycles. In short, based on logging data, the intuitional modes of identifying sedimentary cycles can be obtained through effective processing with geophysical methods. The results of a large number of model experiments have shown that the sedimentary cycles derived by geological methods and by geophysical methods are in good agreement. Our methods were applied to the actual logging data from the Changqing and Dagang Oilfields and received recognition. Now, the methods have been used in other areas.

\subsection{Spectrum analysis}

Spectrum analysis is implemented by transforming a signal in the time domain (or depth domain) to the frequency domain by Fourier transform. In the frequency domain, we can analyze the amplitude and phase of each frequency component, so the method can be applied to indicating different sedimentary cycles. Since the late 1980s, the method has been widely used in the analysis of seismic traces and in the study on the vertical inhomogeneity of stratum structure, the sedimentary rhythm, and the horizontal comparison of thin beds.

In recent years, spectrum analysis has been applied to analyzing the sedimentary cycles caused by global climate change (0.4-1.5, 0.1, 0.02-0.04 Ma) (Li, 1996; Jin and Chen, 2007). For example, previous studies showed that whether the Milankovitch cycle is in the sedimentary records could be concluded from the ratios among different frequency components of Milankovitch cycle, which have been stable over time (Goldhammer et al, 1990; Meyers et al, 2008). At present, this study has been extended to the pre-Quaternary strata, and the feasibility has been proved (Chen and Yang, 1996). Zheng et al (2007) also proved that it was feasible to derive the information of Milankovitch cycles and determine the sedimentary cycles by using the spectrum analysis method, and it can also be used to calculate the deposition rate. Based on previous studies, we used spectrum analysis to distinguish the Milankovitch cycles, and determined the number of the main sedimentary cycles and the thickness ratio of the main cycles in the distinguishable strata. The main cycles were outlined by spectrum graphs to improve the precision of other methods (variable-scale analysis and wavelet depth-frequency analysis). The spectrum analysis method to distinguish sedimentary cycles is only a premise, and it needs to be combined with the transform information from logging data.

In order to identify the internal features and cycle types of formation, we used the spectrum analysis method to process logging data from the Changqing Oilfield, and analyzed the high-level cycles in sedimentary reservoirs. The main study content includes the logging data of Gamma Ray (GR), Spontaneous Potential (SP), and Acoustic log (AC). In practice, gamma logging is sensitive to changes of sedimentary environment, especially in shaly sandstone sequences. Firstly, in an interval, of which the tectonic activity is relatively stable and the sedimentary records are continuous, gamma logging data sampled by a specific step were reconstructed through wavelet analysis, and then the reconstruction data were transformed by FFT. The main frequency components of the analyzed interval can be found, and the ratio of frequency can be obtained, then the main cycles and their thickness ratios can be determined. Under the control of main cycles, we divided and identified all cycles from the curve of the reconstruction data. At present, the spectrum research can only determine the number of cycles in the formation, and it can not be used to determine the specific location of each cycle. Therefore, the curve of the reconstruction data and spectrum must be combined to identify sedimentary cycles more accurately.

We did the spectrum analysis of the well Dan 105 within interval from 1106 to $1510 \mathrm{~m}$ and the well Yuan 296 from 1834 to $2084 \mathrm{~m}$ respectively in the Changqing Oilfield, as shown in Fig. 1(a) and (b). The geological significances of the analysis are as follows: a number of different sedimentary cycles result in a superimposed logging curve which could be decomposed into independent cyclic cycles recorded as frequency spectrum curves through FFT. Low frequency indicates large thickness of the sedimentary cycle, while high frequency indicates a thinner sedimentary cycle. That is to say, the tuning wavelength of the logging signal in thin layers corresponds to the high frequency and that in thick layers corresponds to the low frequency ( $\mathrm{Xu}$ and Wang, 2008). The high-power point in the Fig. 1(a) and (b) indicates the significance of the frequency's sedimentary cycle in the strata. The higher the power, the more the cyclic sedimentary cycles appear in the strata. The frequency of the peak corresponds to the main frequency of the curve, so that the corresponding wavelength and the period of the sedimentary cycle can be obtained.

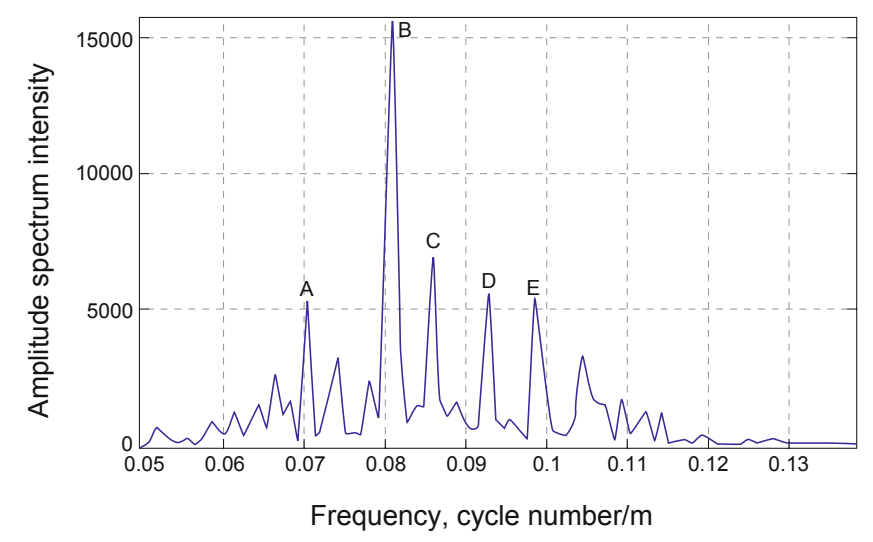

(a) Well Dan 105 


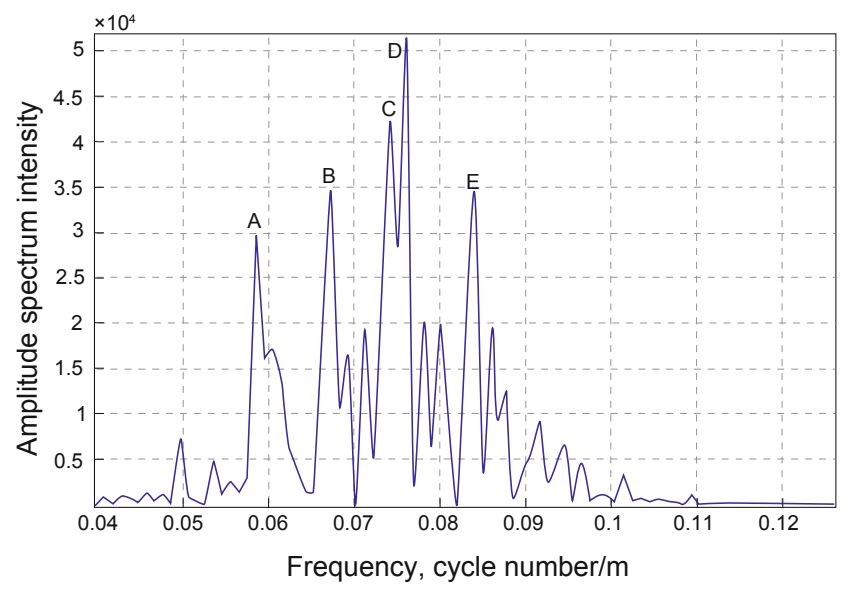

(b) Well Yuan 296

Fig. 1 Spectrum of GR logging curve

From Fig. 1(a), the GR spectrum of the well Dan 105 contains five main frequency points: A, B, C, D, and E with the frequency values of $0.07,0.081,0.0864,0.093$, and 0.099 , respectively, and their tuning thicknesses are 14.29, 12.35, $11.57,10.75$, and $10.10 \mathrm{~m}$, respectively. The sample interval of the curve is $0.125 \mathrm{~m}$, so the main cycle thicknesses of the strata that can be resolved are 1.78, 1.544, 1.446, 1.344, and $1.263 \mathrm{~m}$, with the ratios approximately 1:0.87:0.81:0.76:0.71 (the ratios are used to determine whether the strata contain Milankovitch cycles), and then five cycles can be obtained. How can we determine the interface locations of the five cycles? The curve of the reconstruction data must be used. In Fig. 2, the right spectrum analysis curve is the curve of the reconstruction data that can clearly indicate the interface of each cycle which has large amplitude and high energy value. Fig. 2 showed that the results of spectrum analysis method and those of other methods were in good agreement.

From Fig. 1(b), it can be seen that the GR spectrum of the well Yuan 296 also contains five main frequency points: A, $\mathrm{B}, \mathrm{C}, \mathrm{D}$, and $\mathrm{E}$ with the frequency values of $0.059,0.0675$, $0.0742,0.0758$, and 0.0842 , respectively, and then five cycles in the strata can be obtained. The analysis procedure is the same as that used for well Dan 105. We also got five cycles from the right spectrum analysis curve of Fig. 3, which can clearly indicate the interface of each cycle which has large amplitude and high energy value. Fig. 3 showed that the results of spectrum analysis method and those of other methods were in good agreement.

We used the spectrum analysis method to process data from the Yanchang Group in the study area, and obtained the number and locations of main sedimentary cycles, and the main cycle thickness ratio in the strata that can be resolved. The main cycle thickness ratio is the basis of determining Milankovitch cycles. We applied three methods, spectrum analysis, variable-scale frequency cycle analysis, and wavelet depth-frequency analysis, to the data from well Dan 105 and Yuan 296, and the results were in good agreement, as shown in Fig. 2 and Fig. 3.

\subsection{Variable-scale frequency cycle analysis}

We proposed a new method variable-scale frequency cycle analysis to study sedimentary cycles. The basic idea of logging signal variable-scale analysis is that the signals need to be resolved in different scales. The resolved signals in the large scale are called smooth signals, reflecting the general low-frequency information of logging signals. The signals in the small scale while disappearing in the large scale are called detailed signals, reflecting high-frequency detailed information of logging signals. The geological significances are as follows: Through the wavelet decomposition, the logging curve superimposed by a number of sedimentary cycles with different periods (or scales) is resolved into independent sedimentary cycles, which are presented in the form of scale. The large scale shows that the wavelet is extended. The observation window is spatially large, corresponding to the long period component of signals, which indicates the long period of the deposition and the large thickness of the cycle. This is called large-scale frequency cycle. In other words, the macroscale frequency cycle reflects the large-level formation cycle; the mesoscale frequency cycle reflects the cycle characteristics of the deposition; the microscale frequency cycle reflects the cycle categories within the small sedimentary reservoir, i.e., the small-level high-frequency cycles, as shown in Fig. 2.

The data from the Yanchang Group of well Dan 105 in the Changqing Oilfield were analyzed using the variable-scale frequency cycle method, and the results were shown in Fig. 2 . The method can clearly reflect the cyclicity characteristics of the formation. Large scale corresponds to the frequency cycle characteristics when the scale is equal to 1024 , and can be divided into five sedimentary cycles; mesoscale corresponds to the frequency cycle characteristics when the scale is 512, including nine middle-level sedimentary cycles; small scale corresponds to the frequency cycle characteristics when the scale is 256 , including fifteen small sedimentary cycles. The above mentioned values 1024,512 , and 256 are the scale factors, i.e., the different values of the time window when processing and analyzing the data, and each value is determined based on the mathematical algorithm and geological characteristics. Fig. 2 also marked the lithology columns and the results of wavelet depth-frequency analysis. It is clear that the results of the variable-scale frequency cycle analysis method are consistent with the results from using lithology columns and logging curves, and those of wavelet depth-frequency analysis method, which has proved the effectiveness and accuracy of this method.

\subsection{Wavelet depth-frequency analysis}

Sedimentary studies demonstrate that different rhythm characteristics of sediments form sedimentary cycles of different levels. Logging information is the integrated reflection of the strata medium. Therefore, changes of the property of the strata caused by sedimentary cycles are reflected in the logging information in the depth domain.

We also proposed a new method wavelet depthfrequency analysis to study the sedimentary cycles, and it 


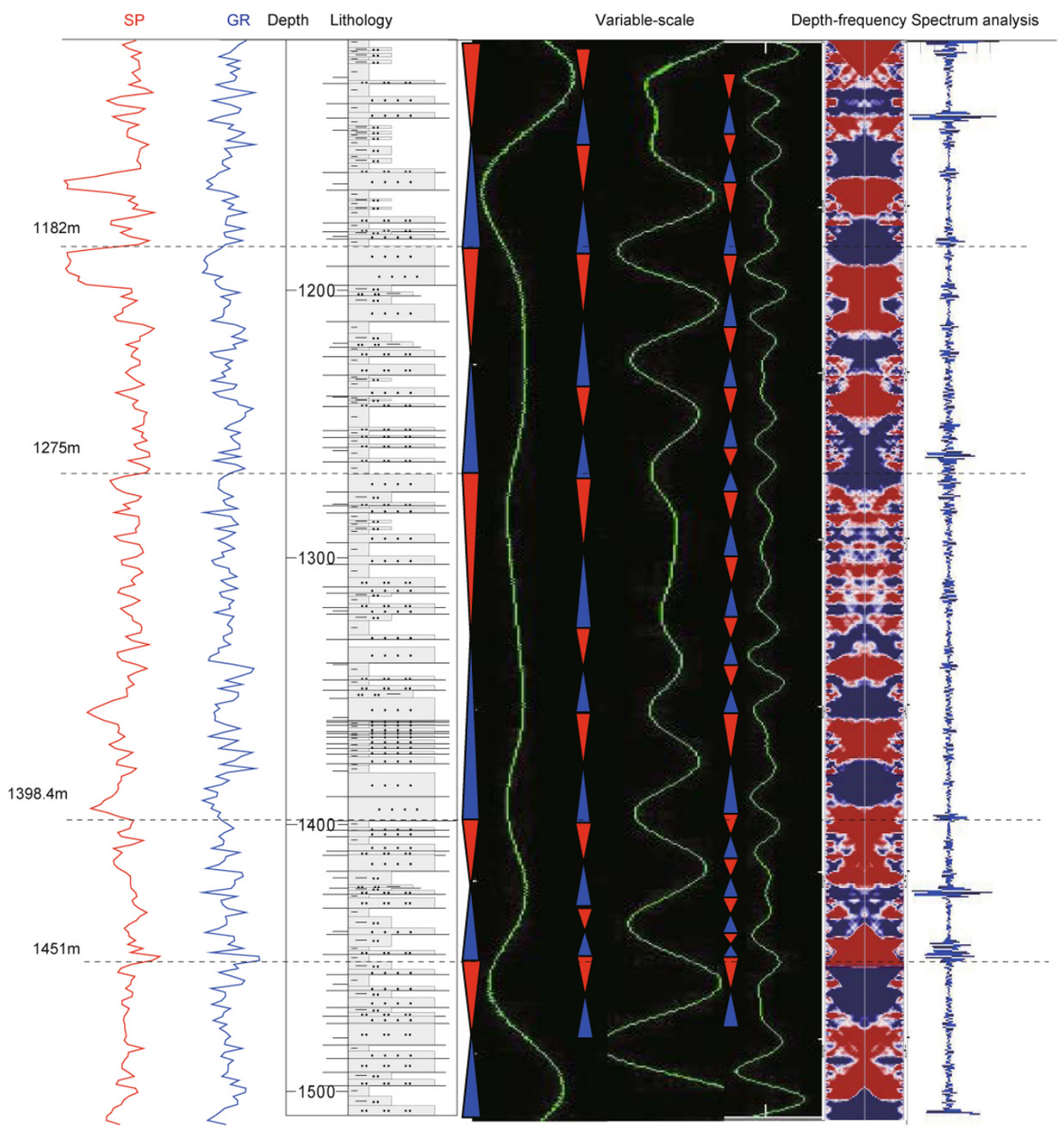

Fig. 2 Variable-scale frequency cycle analysis of GR logging curve of well Dan 105

mainly included two key techniques: frequency division multi-scale automatic processing along layer and highresolution section reconstruction. This method is based on the "magnifying glass" characteristics of the wavelet. The principle is as follows: Through the frequency scanning of the observation records of sedimentary layers, we can find the rule of the main frequency changing with depth and the local characteristics of signals in the depth domain can be described. Because the sedimentary process is periodic, the changes of lithology, grain size composition, and the layer thickness are directional. The directional changes of structure characteristics of thin layers determine the differences of frequency components in physical responses. Therefore, wavelet depth-frequency analysis can use the differences to identify the boundaries of sedimentary cycles of different levels.

We designed a processing software system, which contained the frequency division multi-scale automatic processing along layer and high-resolution section reconstruction and realized the direct identification of sedimentary cycles of different levels from actual logging data. It can clearly identify the large formation cycle, the middle-level sequence, and the cyclicity of depositional bodies, as shown in Fig. 3. It can also clearly identify the rhythm characteristics of the depositional units within oil-bearing layers, that is to say, the resolution of identifying high-frequency sedimentary cycles can achieve the cycle rhythm characteristics within the thin layer, as shown in Fig. 4.

Good results were obtained by using the system to process and analyze the logging data from the Changqing Oilfield. For instance, the wavelet depth-frequency analysis result of well Yuan 296 is shown in Fig. 3. It is shown that this method can identify the large-level formation cycles whose depth is from 1900 to $2012 \mathrm{~m}$, and the interface energy is stronger than that of other sequences and sedimentary cycles. Meanwhile, the cycle interface is very clear. From the bottom to the top of the cycle, the frequency changes from high to low, and then changes from low to high, reflecting a big periodic sedimentary process. 
At the same time, the cycle characteristics of the middle-level sequences and sedimentary bodies are also identified and scaled, and their cycle interfaces are also clear. The frequency also shows the trend of from high to low and then from low to high. The frequency characteristics of sequence cycles reflect rising lake levels, lake flooding, and falling lake levels, i.e., the sedimentary course of retrogradation, aggradation, and progradation. The frequency characteristics of various sedimentary cycles reflect the characteristics of sedimentary microfacies. Fig. 3 also shows the results of variable-scale frequency cycle analysis, which are consistent with those of wavelet depth-frequency analysis.

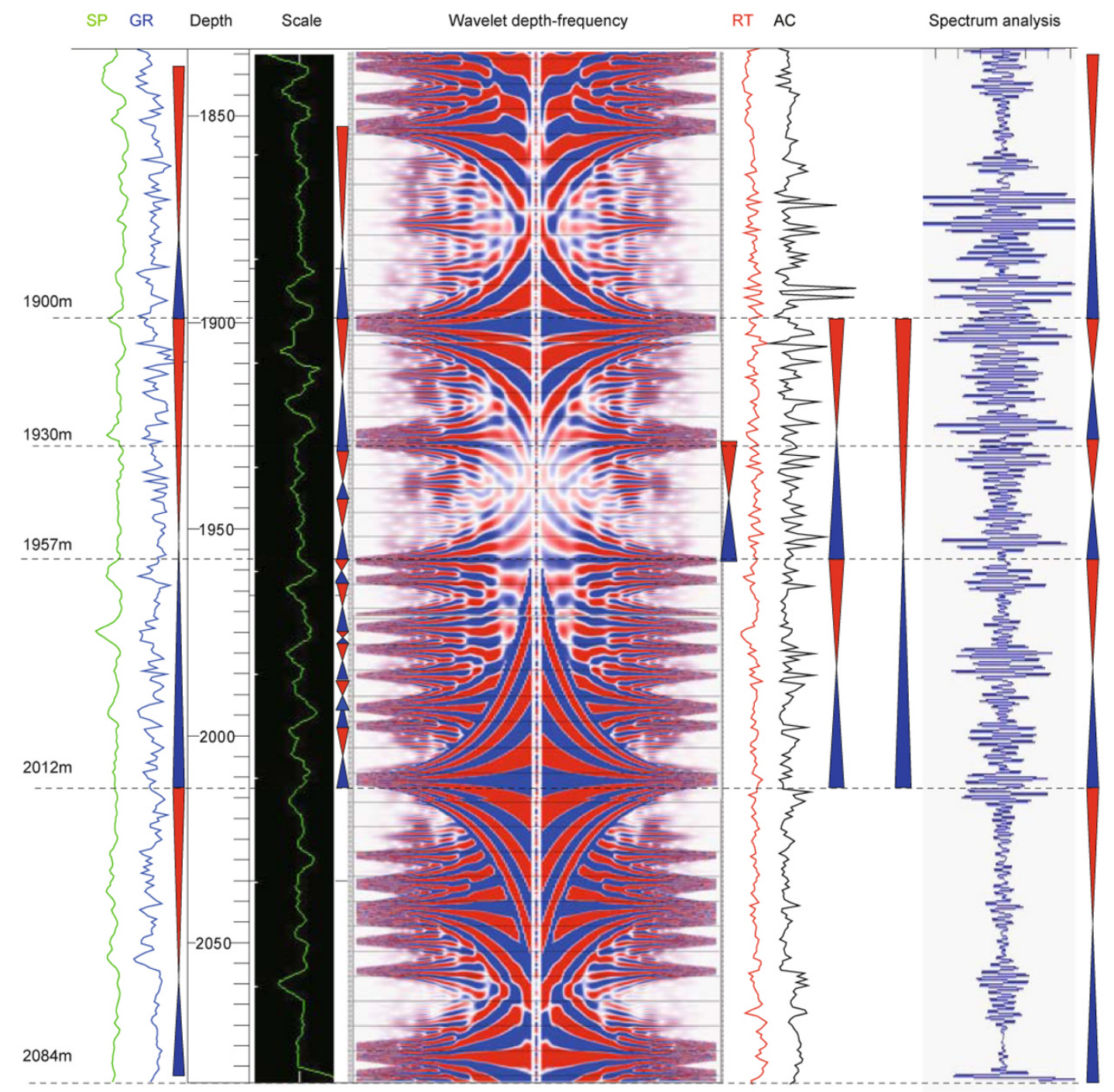

Fig. 3 Wavelet depth-frequency analysis results of well Yuan 296

We processed the actual logging data of well Ning 28 in the Changqing Oilfield by using wavelet depth-frequency analysis to identify and scale the cycles within laminasets and the results were shown in Fig. 4. It can be seen that the method can identify and scale laminasets with clear frequency interfaces and strong energy of cycles. In a single cycle, from bottom to top, the frequency changes from low to high and then from high to low, reflecting the overlapped deposition of sand-mud thin layer within the sedimentary sequence. In the depth from 1788 to $1808 \mathrm{~m}$, two sedimentary sequences are distinguished, which is more precise than previous results.

\section{Conclusions}

By processing and analyzing actual logging data from the
Yanchang Group in the Changqing Oilfield, the following conclusions have been drawn:

1) By analyzing the results of the processed logging data, the feasibility and effectiveness of the three methods to identify sedimentary cycles have been proved and the results of the three methods match very well.

2) Spectrum analysis is a relatively simple method for studying sedimentary cycles. It can be used to calculate the number of main cycles, identify the cycle interfaces, and calculate main cycle thickness ratios of the strata that can be resolved. The cycle thickness ratios are the basis to determine whether the formation contains Milankovitch cycles.

3) In the variable-scale frequency cycle analysis method, different scales are used to distinguish sedimentary cycles 


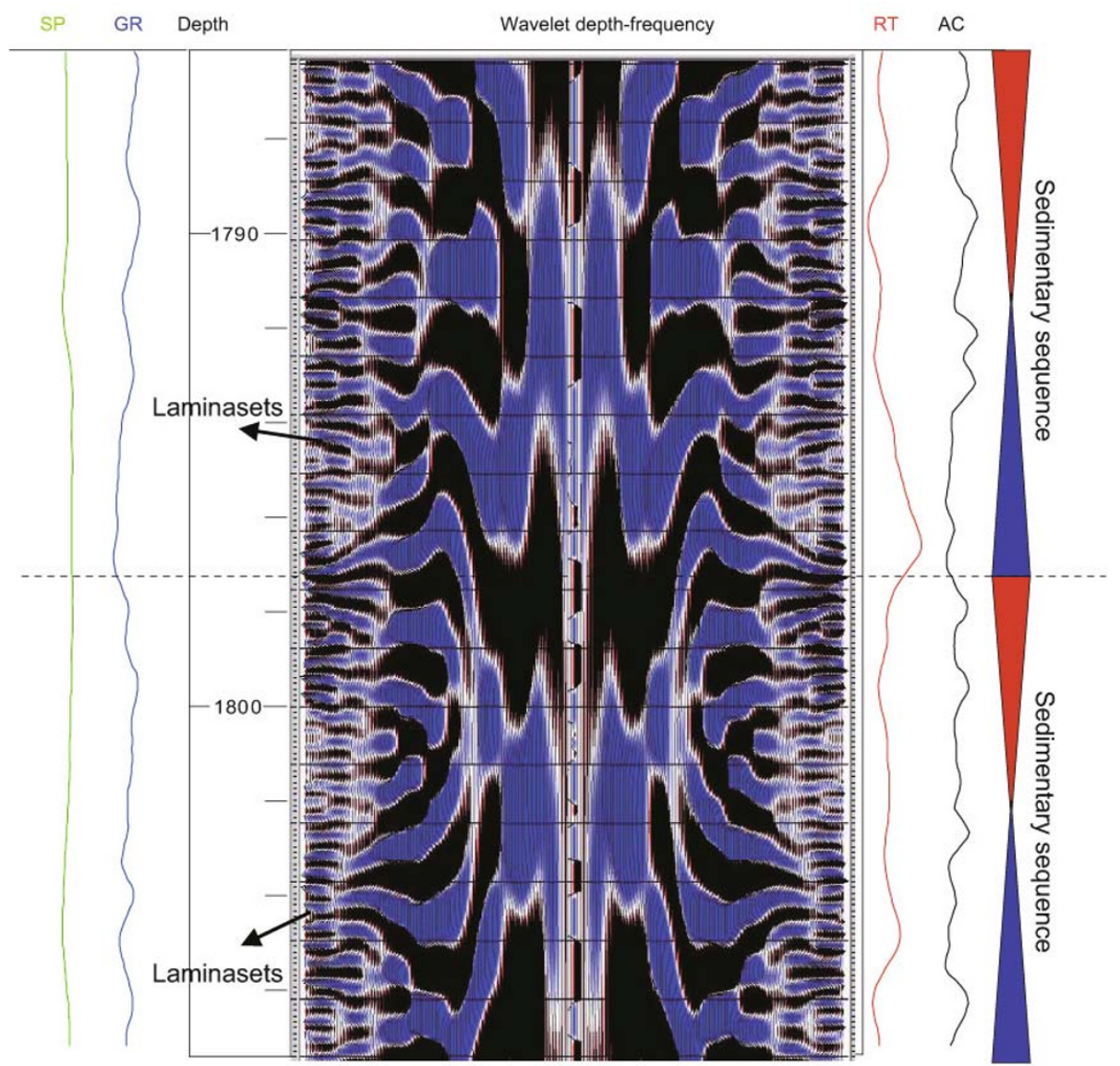

Fig. 4 Wavelet depth-frequency analysis results of well Ning 28

of different levels. When the value of scale is 1024, it can identify large cycles; when the value of scale is 512 , it can identify sedimentary bodies; when the value of scale is 256 , it can identify the rhythm characteristics of sublayers.

4) The wavelet depth-frequency analysis method can identify and scale different cycles from large cycles to small rhythm characteristics. The resolution of this method is relatively high, and different frequency changes reflect different depositional processes.

\section{Acknowledgements}

This work was supported by the National Science \& Technology Major Project (No.2008ZX05020) of CNPC, and we also appreciate the constructive help and encouragement of Dr. Guoping Bai in China University of Petroleum.

\section{References}

Chen G H, Yu J and Zhang X Z. Logging sequence stratigraphic division based on wavelet time-frequency analysis. Xinjiang Petroleum Geology. 2007. 28(3): 355-358 (in Chinese)

Chen Z Q and Yang J G. Records of Milankovitch cycles in prequaternary deposits. Acta Micropalaeontologica Sinica. 1996. 13(1): 65-73 (in Chinese)

Fang W J, Fan Y R, Deng S G, et al. Application of multi-scale analysis to the demarcation of parasequence automatically in well logging. Progress in Geophysics. 2007a. 22(6): 1809-1814 (in Chinese)
Fang W J, Fan Y R, Deng S G, et al. Recognition of parasequences based on wavelet transform of log data. Journal of Stratigraphy. $2007 \mathrm{~b}$. 31(3): 303-308 (in Chinese)

Goldhammer R K, Dunn P A and Hardie L A. Depositional cycles, composite sea-level changes, cycle stacking patterns, and the hierarchy of stratigraphic forcing: Examples from Alpine Triassic platform carbonates. The Geological Society of America Bulletin. 1990. 102(5): 535-562

Jin L Y and Chen F H. Progress in rapid climate changes and their modeling study in millennial and centennial scales. Advances in Earth Science. 2007. 22(10): 1054-1065 (in Chinese)

Li F J, Wang D Y, Zheng X M, et al. The application of frequency spectral analysis of logging curves on sedimentary cycle studies in coal-bearing strata. Coal Geology \& Exploration. 2003. 31(6): 14-18 (in Chinese)

Li Q M. The analysis and application of Milankovitch cycles by logging data. Chinese Journal of Geophysics. 1996. 39(5): 699-704 (in Chinese)

Liu B and Fang W J. Application of multiscale analysis to logging data processing. Journal of Shandong Jianzhu University. 2006. 21(6): 549-552 (in Chinese)

Liu G D. Discussion on earth sciences. Earth Science Frontiers. 1998. 5(1): 1-8 (in Chinese)

Li X H. Sequence stratigraphic analysis with curve inflexion from geophysical logging data. Natural Gas Geoscience. 2006. 17(6): 815819 (in Chinese)

Meyers S R, Sageman B B and Pagani M. Resolving Milankovitch: consideration of signal and noise. American Journal of Science. 2008. 308(3): 770-786 
Peng Y H. Wavelet Transform and Project Application. Beijing: Science Press. 1999. 4-35 (in Chinese)

Wang G W, Deng Q P and Tang W Q. The application of spectral analysis of logs in depositional cycle studies. Petroleum Exploration and Development. 2002. 29(1): 93-95 (in Chinese)

Xu J L and Wang G W. The application of wavelet depth-frequency analysis to the study of sedimentary cycles. The Fifth Youth Academic Annual Congress of China Petroleum Society. Dongying:
China University of Petroleum Press. 2008. 138-150 (in Chinese) Zhao J L and Li N. Application of wavelet transform to high resolution sequence analysis. Progress in Geophysics. 2008. 23(4): 1230-1235 (in Chinese)

Zheng M, Peng G X, Lei G L, et al. Applying frequency spectral analysis to identify Milankovitch cycle and calculate sedimentation rate of cretaceous in Wushi Sag. Xinjiang Petroleum Geology. 2007. 28(2): 170-174 (in Chinese)

(Edited by Hao Jie) 